\title{
Seraph-100 Hemoperfusion in SARS-CoV-2-Infected Patients Early in Critical Illness: A Case Series
}

\author{
Brian S. Rifkin ${ }^{a} \quad$ lan J. Stewart ${ }^{\mathrm{b}}$ \\ ${ }^{a}$ Hattiesburg Clinic Department of Nephrology, Hattiesburg, MS, USA; ${ }^{b}$ Department of Medicine, Uniformed \\ Services University, Bethesda, MD, USA
}

\section{Keywords}

Coronavirus disease 2019 - Extracorporeal sorbent hemoperfusion · Seraph-100 · Critical illness · Case report

\begin{abstract}
There is an urgent need for therapeutic interventions to alter the course of critically ill coronavirus disease 2019 (COVID-19) patients. We report our experience with the Seraph-100 Microbind Affinity Blood Filter (Seraph-100) in 4 patients with COVID-19 early in the course of their critical respiratory illnesses. Patients were diagnosed with COVID-19 and were admitted to intensive care with worsening respiratory failure but did not require dialysis or vasopressors. Patients had to have $\mathrm{PaO}_{2}$ to $\mathrm{FiO}_{2}(\mathrm{P} / \mathrm{F}$ ratio) $<150$ to qualify for hemoperfusion therapy. All patients received standard medical therapy including oral vitamins $C$ and $D$ and zinc in addition to intravenous dexamethasone and remdesivir. Patients received a single 5- to 7-h session with Seraph-100 on a conventional dialysis machine (Fresenius 2008T) via a nontunneled central venous dialysis catheter with a goal of processing at least $100 \mathrm{~L}$ of blood. Patients received weight-based subcutaneous enoxaparin anticoagulation, as well as systemic intravenous heparin (70 units/kg), just prior to hemofiltration. Treatment with Seraph-100 hemoperfusion was
\end{abstract}

well tolerated, and all patients were able to finish their prescribed therapy. All patients treated with Seraph-100 survived to be discharged from the hospital. Well-designed clinical trials are needed to determine the overall safety and efficacy of the Seraph-100 Microbind Affinity Blood Filter in COVID-19 patients.

(c) 2021 S. Karger AG, Basel

\section{Introduction}

SARS-CoV-2, coronavirus disease 2019 (COVID-19), has killed millions of people world-wide [1] and put enormous strain on the health-care system. Unfortunately, few interventions have consistently been shown to improve patient outcomes. Mortality has been noted to approach $88 \%$ [2-4] among intubated patients admitted to the ICU.

One potential therapy for treating critically ill COVID-19 patients is extra-corporeal blood purification (ECBP). ECBP is currently an unvalidated and unproven therapy, but it has been theorized to be potentially beneficial in the treatment of infectious diseases. A new ECBP device, the Seraph-100 Microbind Affinity Blood Filter (ExThera Medical Corporation, Martinez, CA, USA), 
Table 1. Clinical characteristics of patients treated with Seraph-100 hemoperfusion filter

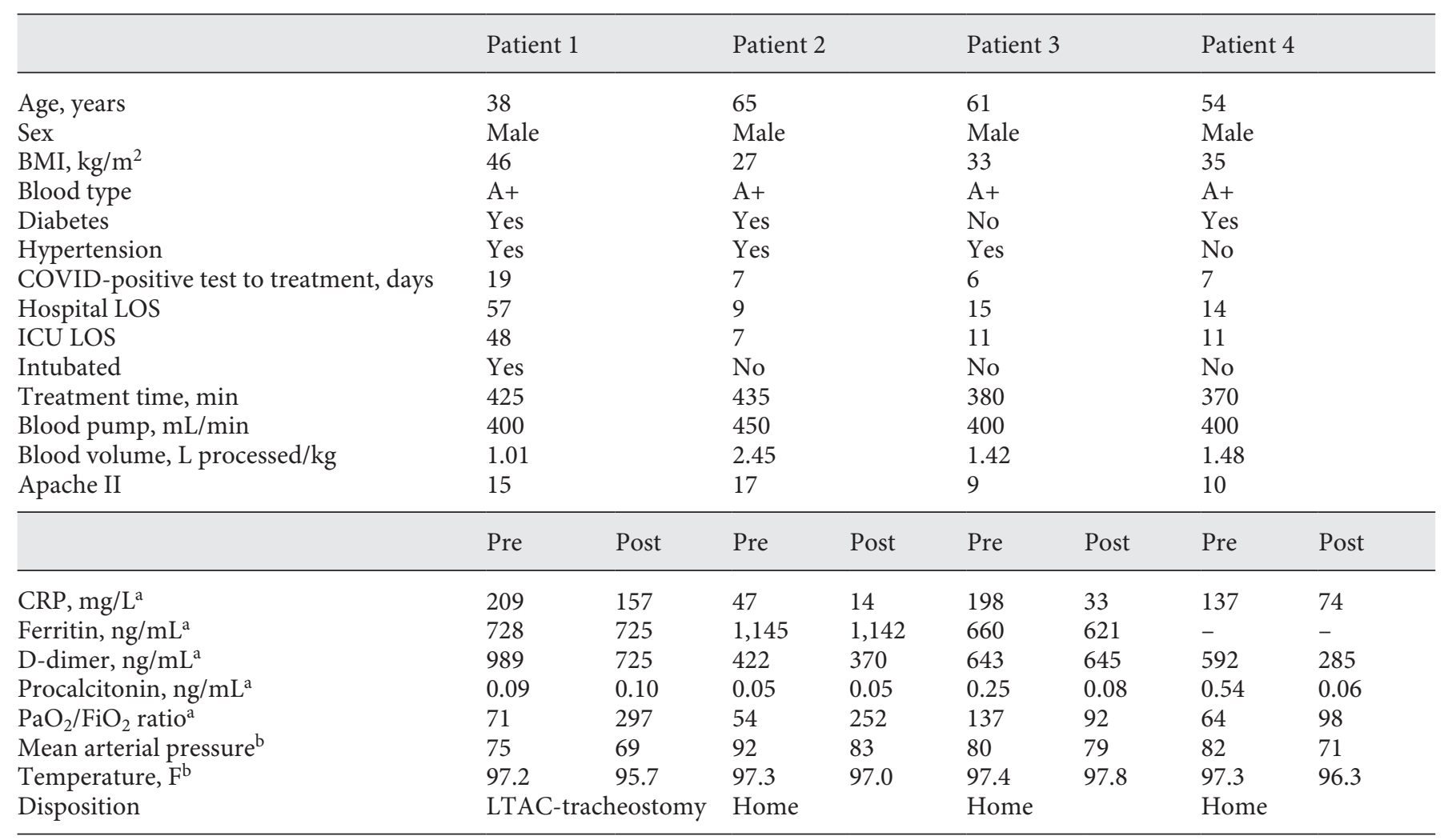

${ }^{a}$ Values obtained between 6 and $12 \mathrm{~h}$ before and after Seraph treatment. ${ }^{\mathrm{b}}$ Values obtained within $1 \mathrm{~h}$ of start/finish of Seraph treatment.

composed of heparin bound to polyethylene beads became available in the United States under Emergency Use Authorization for patients with COVID-19. This system has been shown to bind a wide range of pathogens, including both bacteria and viruses [5].

\section{Case Summaries}

All patients were admitted to the medical intensive care unit with a PCR diagnosis of COVID-19 and acute respiratory distress syndrome with a $\mathrm{PaO}_{2}$ to $\mathrm{FiO}_{2}(\mathrm{P} / \mathrm{F})$ ratio $<150$. In this series, none of the patients required renal replacement therapy or treatment with vasopressors during their ICU admissions. All patients received standard therapy including oral vitamins $C$ and $\mathrm{D}$ and zinc along with intravenous dexamethasone and remdesivir. All patients had blood, urine, and sputum cultures on admission to the ICU, none of which indicated a concomitant bacterial infection. Patient characteristics are summarized in Table 1.

\section{Discussion}

In this single-center case series of SARS-CoV-2-infected patients, we witnessed clinical improvement in patients treated with Seraph-100. Based on a scoring system for mortality in hospitalized COVID-19 patients [6], all our patients were "high risk" with mortality estimates between 31.4 and $34.9 \%$. We found that Seraph-100 was safe and well tolerated. The expected complications with ECBP are similar to those seen with hemodialysis. Associated risks include bleeding, clotting, infections related to central venous catheter use, filter sensitivity reactions, and hypotension. Treatments were well tolerated, and patients were not noted to experience any complications. All Seraph-100 treatments were performed on a conventional dialysis platform (Fresenius 2008T) in series with a hemodialysis filter. Patients received intravenous heparin (70 units $/ \mathrm{kg}$ ) at the start of treatment to prevent clotting of the blood lines, dialysis circuit, and Seraph-100 filters. No hemodialysis was performed, as the dialysis bath was 
Fig. 1. Seraph-100 hemoperfusion filter setup on a conventional dialysis machine.

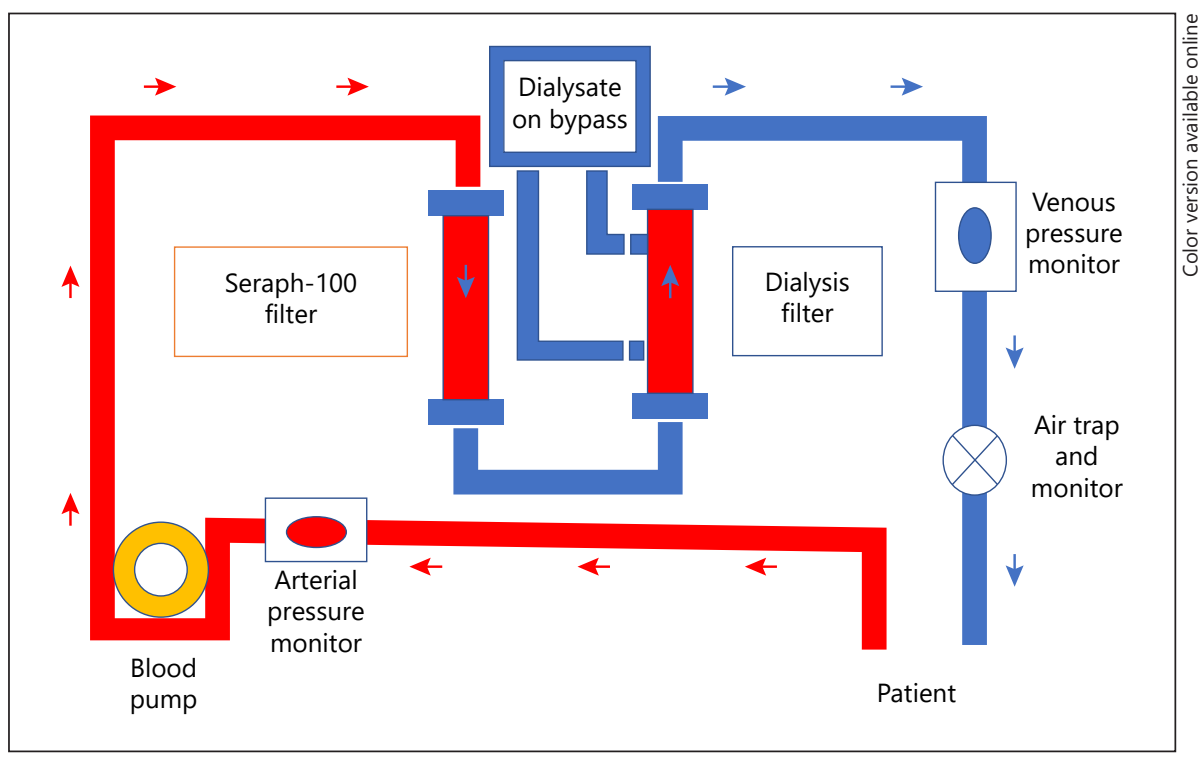

placed on bypass. The series of filters was required because we were not able to perform isolated hemofiltration with our dialysis machine. We were, however, able to make use of the dialysis machine's blood pump and safety features with the configuration shown in Figure 1. Of note, we observed that 3 out of the 4 patients treated with Seraph-100 had improvements in their P/F ratios. This has also been observed in 1 other case report [7] and in unpublished data [8]. While the mechanism of action for this improvement is unclear, we hypothesize that Seraph-100 may have improved endothelial function via its resemblance to the glycocalyx.

Olson et al. [9] previously reported a case series of 2 critical patients on mechanical ventilation and vasopressors treated with Seraph-100. High-level viremia has been associated with pro-inflammatory cytokines and increased mortality [10]. Seraph-100 works by directly binding the coronavirus, and thus, we hypothesize treatment early in the course of critical illness may attenuate the subsequent hyper-inflammatory response. In our experience, early treatment may have prevented further decline and the need for mechanical ventilation in 3 patients.

\section{Conclusion}

We present a case series of 4 COVID-19 patients treated with the Seraph-100 hemoperfusion device in the ICU. Patients were early in the course of their disease and did not require vasopressors or renal replacement. We used Seraph-100 early to try to prevent intubation and worse clinical outcomes. All 4 patients in our series appeared to improve after initiation of therapy. All patients ran for their prescribed time, and no complications were noted. Additional studies are needed to clarify the ideal timing for initiation, length of treatment, and if consecutive treatments are required to achieve the best possible patient outcomes. Randomized controlled trials are needed to establish safety and efficacy prior to the wide-spread adoption of Seraph-100 for the treatment of COVID-19.

\section{Disclaimer}

The opinions and assertions expressed herein are those of the author(s) and do not necessarily reflect the official policy or position of the Uniformed Services University or the Department of Defense.

Identification of specific products or scientific instrumentation is considered an integral part of the scientific endeavor and does not constitute endorsement or implied endorsement on the part of the authors. The authors have no conflicts to declare.

\section{Statement of Ethics}

Research complied with the guidelines for human studies and was conducted ethically in accordance with the World Medical Association Declaration of Helsinki. A central IRB approved the study protocol for use at multiple US-based hospital sites. Written informed consent was obtained by the patients for publication of this case report and any accompanying images published. 


\section{Conflict of Interest Statement}

The authors have no conflict of interest to declare.

\section{Funding Sources}

Seraph-100 devices were provided free of charge by the manufacturer (ExThera Medical Corporation, Martinez, CA, USA).

\section{Author Contributions}

Dr. Rifkin was involved with patient recruitment, data gathering, and writing of the manuscript. Dr. Stewart was involved in writing and editing of the manuscript.

\section{References}

1 Johns Hopkins University. Coronavirus Resource Center [cited 2020 Dec 29]. Mortality Analysis. Available from: https://coronavirus. jhu.edu/data/mortality.

2 Bhatraju PK, Ghassemieh BJ, Nichols M, Kim R, Jerome KR, Nalla AK, et al. Covid-19 in critically ill patients in the Seattle region: case series. N Engl J Med. 2020;382(21):2012-22.

3 Huang C, Wang Y, Li X, Ren L, Zhao J, Hu Y, et al. Clinical features of patients infected with 2019 novel coronavirus in Wuhan, China. Lancet. 2020;395:497-506.

4 Richardson S, Hirsch JS, Narasimhan M, Crawford JM, McGinn T, Davidson KW, et al. Presenting characteristics, comorbidities, and outcomes among 5700 patients hospitalized with COVID-19 in the New York city area. JAMA. 2020;323:2052-9.
5 Seffer MT, Cottam D, Forni LG, Kielstein JT. Heparin 2.0: a new approach to the infection crisis. Blood Purif. 2021;50(1):28.

6 Knight SR, Ho A, Pius R, Buchan I, Carson G, Drake TM, et al. Risk stratification of patients admitted to hospital with covid-19 using ISARIC WHO clinical characterization protocol: development and validation of the $4 \mathrm{C}$ mortality score. BMJ. 2020;370:m3339.

7 Seffer MT, Eden G, Engelmann S, Kielstein JT. Elimination of Staphylococcus aureus from the bloodstream using a novel biomimetic sorbent haemoperfusion device. BMJ Case Rep. 2020;13:e235262.
8 Buttner S. Extracorporeal pathogen elimination is on the horizon. Presented at the International Symposium on Intensive Care \& Emergency Medicine; 2019.

9 Olson SW, Oliver JD, Collen J, Bunin J, Gleeson TD, Foster BE, et al. Treatment for severe coronavirus disease 2019 with the Seraph-100 microbind affinity blood filter. Crit Care Explor. 2020;2(8):e0180.

10 Fajnzylber J, Regan J, Coxen K, Corry H, Wong C, Rosenthal A, et al. SARS-CoV-2 viral load is associated with increased disease severity and mortality. Nat Commun. 2020; 11:5493. 